\title{
Variation des ressources collectives et organisation des activités de violence au sein du Mouvement nationaliste en Turquie (1975-1980)
}

Variation of collective resources and organization of violent activities in the Turkish Nationalist Movement (1975-1980)

\section{Benjamin Gourisse}

\section{OpenEdition Journals}

\section{Édition électronique}

URL : http://journals.openedition.org/conflits/18104

DOI : $10.4000 /$ conflits. 18104

ISSN : $1777-5345$

\section{Éditeur :}

CCLS - Centre d'études sur les conflits lilberté et sécurité, L'Harmattan

\section{Édition imprimée}

Date de publication : 5 septembre 2011

Pagination : 81-100

ISBN : 978-2-296-56086-4

ISSN : $1157-996 \mathrm{X}$

\section{Référence électronique}

Benjamin Gourisse, «Variation des ressources collectives et organisation des activités de violence au sein du Mouvement nationaliste en Turquie (1975-1980) », Cultures \& Conflits [En ligne], 81-82 | Printemps/Été 2011, mis en ligne le 05 septembre 2012, consulté le 30 mars 2021. URL : http:// journals.openedition.org/conflits/18104; DOI : https://doi.org/10.4000/conflits.18104 


\section{Variation des ressources collectives et organisation des activités de violence au sein du Mouvement nationaliste en Turquie (1975-1980)}

\section{Benjamin GOURISSE}

Benjamin Gourisse est l'auteur d'une thèse intitulée L'État en jeu. Captations des ressources et désobjectivation de l'État en Turquie (1975-1980). Il étudie les activités de captation des ressources étatiques déployées par les partis politiques, ainsi que leurs effets sur les règles formelles et informelles des jeux politiques et étatiques en Turquie. Dans le cadre de l'ANR Transtur "Ordonner et transiger : modalités de gouvernement et d'administration en Turquie et dans l'Empire ottoman, $d u$ $X I X^{e}$ siècle à nos jours ", il étudie les relations de concurrence institutionnelle et politique internes au champ étatique et les processus de politisation des pratiques des usagers de la fonction publique. Il a notamment publié un article intitulé "Enquête sur les relations entre politisation et études supérieures : le cas turc (1971-1980)" in Aït-Aoudia M., Bennani Chraibi M. et Contamin J.-G., dossier "Politisation des individus et des groupes ", Critique Internationale, n50, janvier-mars 2011.

$\mathrm{E}$ ntre 1975 et 1980, les militants du Mouvement nationaliste ${ }^{1}$ développent tout un ensemble d'activités faisant intervenir la violence physique ${ }^{2}$. Profitant des dysfonctionnements des institutions étatiques de coercition ${ }^{3}$ et de sa participation à plusieurs gouvernements de coalition ${ }^{4}$, le Parti de l'action nationaliste (Milliyetçi Hareket Partisi, MHP, extrême droite nationa-

1. Le Mouvement nationaliste désigne l'ensemble des organisations fondées afin de soutenir l'action du Parti de l'action nationaliste dans sa volonté d'accès au pouvoir. Sa forte centralisation autour du parti permet de le caractériser comme une entreprise tentaculaire d'accumulation et d'allocation de ressources visant à soutenir le parti dans sa stratégie d'accès au pouvoir, et à permettre à ses membres locaux d'accéder aux ressources municipales, et plus largement locales. Dans l'article, nous utiliserons également le terme indigène d' "idéaliste »

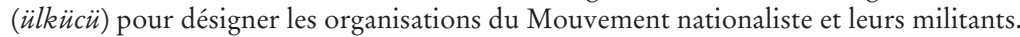

2. La réalisation de l'article a bénéficié du soutien du projet ANR Transtur (Ordonner et transiger : modalités de gouvernement et d'administration en Turquie et dans l'Empire Ottoman du XIX siècle à nos jours).

3. Gourisse B., «Pluralité des rapports aux normes professionnelles et politisation des pratiques dans la police turque des années 1970 », European Journal of Turkish Studies [Online], n8, 2008, URL : http://ejts.revues.org/index2273.html. 
liste), qui est à l'origine de la constitution du Mouvement, met en place une stratégie d'affrontement idéologique et physique systématique avec les groupes d'extrême gauche, dont la base sociale connaît une croissance inédite. Il joue alors d'une tactique tantôt légale, tantôt illégale d'accès au pouvoir en participant au jeu électoral tout en s'engageant dans une guerre civile larvée avec les organisations radicales de gauche. La militarisation des répertoires d'actions de secteurs de ces deux ensembles provoque une augmentation rapide de la violence physique, décelable notamment par l'accroissement du nombre de morts, qui passe de 3 en 1974 à 1368 en 1979 et 1939 pour les neufs premiers mois de l'année 1980, précédant l'intervention militaire du 12 septembre ${ }^{5}$.

Les activités de violence mises en œuvre par les groupes radicaux ne relèvent pas toutes des mêmes logiques. Pendant la seconde moitié des années soixante-dix, les altercations entre militants idéalistes et d'extrême gauche se multiplient. Mais à côté d'une violence « contextuelle » n'exigeant qu'un niveau minimal d'organisation et résultant des rencontres fortuites et des interactions quotidiennes des militants idéalistes et d'extrême gauche, des activités de violence physique organisées et régulières sont repérables. Des " équipes de frappe » (vurucu timler) sont constituées par des sections du MHP ou des organisations idéalistes sur certaines parties du territoire, nécessitant le recrutement ou la formation d'activistes. En outre, les logiques de la mise en œuvre des activités de violence physique sont différentes dans les deux camps en présence. Alors qu'à l'extrême gauche, la violence n'est que peu corrélée aux autres activités des organisations, et se révèle souvent être le fait de groupes autonomisés des organisations mères, celle-ci s'inscrit dans la continuité directe des activités conventionnelles du Mouvement nationaliste. Ainsi, c'est lorsque le MHP dispose d'un accès au gouvernement et parvient à intervenir dans les procédures de nomination dans la fonction publique - et notamment dans la police et les institutions judiciaires - que les activistes du Mouvement parviennent le plus aisément à mettre en œuvre leurs activités de violence.

C'est à l'étude du travail d'encadrement de ces activités de violence organisée par le Mouvement nationaliste qu'est consacré le présent article. Il étudie les dispositifs élaborés par le Mouvement entre 1975 et 1980 afin d'organiser les activités de violence, et leurs transformations dans un contexte de modification des types et des niveaux de ressources collectives. Pour ce faire, nous nous interrogeons sur la façon dont la variation des ressources mobilisables par les organisations du Mouvement, selon que le MHP dispose ou non

4. Gourisse B., "Party Strategies for Investing in State Institutions: MHP in the late 1970s", in Massicard E., Watts N., Breaking Up the Party. Political Parties and Social Forces in Turkey, à paraître.

5. Bozarslan H., « Le phénomène milicien, une composante de la violence politique dans la Turquie des années $1970 »$, Turcica, vol. 31, 1999, pp. 185-244. 
d'un accès aux positions étatiques, modifie les types et l'efficacité de ces dispositifs ${ }^{6}$.

L'observation des modalités de l'usage de la violence par les idéalistes permet alors d'apporter une contribution à l'analyse des déterminants du recours à l'action violente. Elle autorise également à rompre avec les analyses existantes sur la crise politique turque de la seconde partie des années soixantedix.

Il convient d'abord d'abandonner toute conception « mécaniste » de la formation et des modifications des répertoires d'action, liant l'utilisation de la violence aux positions de marginalité, ou à la radicalisation idéologique. Si l'utilisation de modes d'action violents a souvent été associée aux positions et aux niveaux de ressources des groupes mobilisés ${ }^{7}$, c'est généralement pour la considérer comme un moyen susceptible de compenser le faible niveau de ressources du groupe ou de forcer l'accès à la reconnaissance de revendications jusqu'alors ignorées par le système ${ }^{8}$. C'est cette position qu'adopte Charles Tilly lorsqu'il lie l'utilisation de la violence à la position de challenger 9 . L'observation des activités de violence au sein du Mouvement nationaliste tend à réfuter ces analyses, puisque c'est lorsque le MHP accède aux pratiques de nomination dans la fonction publique que les sections idéalistes locales passent le plus souvent à l'action - les soutiens qu'elles trouvent auprès des organes déconcentrés de l'État leur permettant de tenter le «coup de force » localement. On constate alors une continuité entre les activités de violence et les autres, plus conventionnelles, mises en œuvre par le Mouvement. Légal et illégal, étatique et criminel s'enchevêtrent et s'interpénètrent, puisque l'usage de la violence est autorisé par l'ensemble des autres activités et des positions notamment étatiques - des groupes. Il y a alors lieu de renverser cette perspective dominante liant le faible niveau de ressources et le passage à la violence, et de considérer les positions étatiques occupées comme des ressources mobilisables dans l'organisation des activités de violence. En ce sens, notre enquête s'inscrit dans une série de travaux attentifs aux « interactions entre des déten-

6. Entre mars 1975 et janvier 1978, le MHP participe à deux gouvernements de coalition et dispose de possibilités d'intervenir dans les procédures de recrutement de la fonction publique pour y faire recruter ses militants et profiter de leurs activités afin d'assurer un niveau d'impunité élevé à ses activistes. Entre janvier 1978 et novembre 1979, il est dans l'opposition au gouvernement de gauche du Parti républicain du peuple (Cumburiyet Halk Partisi, CHP) et doit s'appuyer sur ses ressources propres afin de mettre en œuvre sa tactique tantôt légale, tantôt illégale d'accès au pouvoir. Enfin, entre novembre 1979 et le coup d'État du 12 septembre 1980, il apporte depuis l'Assemblée un soutien conditionnel à un gouvernement minoritaire de centre droit et gagne de cette façon une influence dans l'État.

7. Zald M. N., "Looking Backward to Look Forward: Reflections on the Past and Future of the Resource Mobilization Research Program", in Morris A. D., Mc Clurg Mueller C. (eds.), Frontiers in Social Movement Theory, New Haven, Yale University Press, 1992, pp. 326-346.

8. Piven F. F., Cloward R. A., Poor People's Movements: Why they Succeed, How they Fail, New York, Vintage, 1977.

9. Tilly C., From Mobilisation to Revolution, Reading, Addison-Wesley Publishing Company, 1978. 
teurs du pouvoir politique et des professionnels de l'usage de la force et de l'intimidation 10 ». En outre, la violence n'apparaît pas, dans le cas étudié ici, comme un produit automatique d'une radicalisation idéologique des individus, mais comme un mode d'action dont l'activation est autorisée par les ressources et les tactiques d'accumulation des groupes.

L'observation de la continuité entre les différentes activités du Mouvement conduit également à rompre avec les analyses produites sur la crise turque de la seconde partie des années soixante dix. Cette crise se caractérise par une multiplication des mobilisations sociales et politiques, animées par l'extrême gauche et le mouvement nationaliste, dont les interactions provoquent une augmentation régulière de la violence jusqu'au coup d'État militaire du 12 septembre 1980. Les assassinats, attentats et fusillades deviennent quotidiens. La situation présente ainsi quelque similitude avec celle que décrit Sebastian Haffner à propos de la République de Weimar au lendemain des élections du 14 septembre 1930 quand il évoque un "état intermédiaire entre "l'ordre et la paix" et la "guerre civile" (il n'y avait pas de barricades, mais il y avait toujours des bagarres, des fusillades [...], des attentats dirigés contre les locaux des divers partis, et sans cesse de nouveaux morts) 11 ». Dans ce contexte, l'État semble incapable de rétablir l'ordre, tant il est exposé aux tactiques de pénétration menées par ces organisations radicales. La grande majorité des analyses produites sur la crise turque repose sur les trois postulats que sont la symétrie des ressources et des positions de l'extrême gauche et du Mouvement nationaliste, la faillite d'un État se retrouvant hors-jeu 12 consécutivement à leurs mobilisations, et l'autonomisation de la violence, identifiable par la formation d'une conjoncture anarchique ou prérévolutionnaire ${ }^{13}$. Cependant, l'observation donne à voir une forte asymétrie des ressources en faveur des idéalistes qui bénéficient de l'accès au gouvernement du MHP et des positions étatiques qu'il parvient à leur assurer. L'État n'est donc pas hors-jeu, puisque les positions et les ressources qu'il offre servent les activités des groupes mobilisés. Dans ce contexte, l'usage de la violence par le Mouvement nationaliste doit être étudié comme un aspect de stratégies plus larges, et son analyse doit être intégrée à celle de l'ensemble des activités idéalistes. La violence ne s'autonomise alors que marginalement, parce que ses ini-

10. Briquet J-L., Favarel-Garrigues G. (eds.), Milieux criminels et pouvoir politique. Les ressorts illicites de l'État, Paris, Karthala, 2008, p. 9. Sur le continuum existant entre mobilisations violentes et conventionnelles, voir également Collovald A., Gaïti B. (eds.), La Démocratie aux extrêmes. Sur la radicalisation politique, Paris, La Dispute, 2006 ; McAdam D., Tarrow S., Tilly C., Dynamics of contention, Cambridge, Cambridge University Press, 2001.

11. Haffner S., Histoire d'un Allemand. Souvenirs (1914-1933), Paris, Actes Sud, Babel, 2003, p. 141.

12. Birand M. A., Bilâ H., Akar R., 12 Eylül. Türkiye'nin miladı (12 septembre. La nouvelle ère de la Turquie), Istanbul, Do an Kitap, 2006, p. 122.

13. Dans un article consacré à la configuration sociopolitique turque de la seconde partie des années 1970, Hamit Bozarslan a démontré les impasses des analyses faisant de la période un contexte de crise, ou de chaos généralisé : Bozarslan H., « Le chaos après le déluge ? Notes sur la crise turque des années 70 », Cultures E Conflits, n²4-25, 1996-1997, pp. 73-97. 
tiateurs sont liés au champ étatique par les liens collusifs qui les connectent aux secteurs fonctionnarisés du Mouvement nationaliste.

La problématique adoptée tient compte des types de sources disponibles et des difficultés d'accès à la parole des acteurs. Les données mobilisables pour l'enquête renseignent sur l'organisation du Mouvement plutôt que sur les profils et trajectoires de ses membres. Elles autorisent donc l'observation des dispositifs collectifs permettant le passage aux activités de violence, plutôt que celle des carrières activistes elles-mêmes. Des sources écrites permettent ainsi de saisir la façon dont ces dispositifs collectifs servent de médiation pour l'entrée et le maintien dans des carrières activistes. Il s'agit, en premier lieu, du réquisitoire du procureur militaire du commandement de l'état de siège des provinces d'Ankara, Çankırı et Kastamonu au procès du MHP et des institutions idéalistes, tenu à partir du 29 avril $1981{ }^{14}$. Aucun travail de recherche publié à ce jour n'a encore analysé ce document de façon exhaustive, bien qu'il constitue la source la plus riche pour étudier la façon dont le MHP et les organisations idéalistes ont planifié et organisé des activités de violence physique dans les années soixante dix. Des précautions doivent bien sûr être respectées dans l'utilisation des données du réquisitoire, afin de ne pas importer dans le champ de la production scientifique les arguments des institutions de justice militaire. On ne saurait trop se méfier d'une production émanant d'un tribunal dont l'action visait à la fermeture d'un parti identifié comme l'un des principaux acteurs des troubles ayant mené à l'intervention militaire du $12 \mathrm{sep}-$ tembre 1980 15. D’autres sources permettent parfois de vérifier les allégations du procureur, de les compléter ou de les corriger. Nous avons ainsi mené un travail de comparaison et de vérification systématique des informations proposées par le réquisitoire. Le dépouillement de deux des plus grands quotidiens nationaux de l'époque, Cumburiyet et Milliyet, parus entre le premier janvier 1977 et le 31 décembre 1980, a ainsi permis de corroborer ou d'invalider les informations proposées par le procureur. Des mémoires 16 et des témoignages 17 d'activistes idéalistes, publiés dans les années soixante dix à la suite d'arrestations ou de repentances, nous ont également aidé à repérer les

14. T. C. Ankara - Çankırı - Kastamonu İlleri Sıkıyönetim Komutanlığı Askeri Sacılı̆̆ı. Iddianame : MHP ve Ülkücü Kuruluşlar (Procureur militaire du commandement de l'état de siège des provinces d'Ankara - Çankir - Kastamonu. Réquisitoire : Le MHP et les organisations idéalistes), préparé par le procureur militaire de l'État de siège Nurettin Soyer, 1981.

15. Pour une analyse critique des relations entre pouvoir politique et pouvoir judiciaire, voir Briquet J-L., Mafia, justice et politique en Italie. L'affaire Andreotti dans la crise de la République (1992-2004), Paris, Karthala, 2007.

16. Tanlak Ö., Itiraf. Eski bir ülkücü MHP'yi anlatzyor (Confession. Un ancien idéaliste explique le $M H P)$, Istanbul, Kaynak Yayınları, 1996.

17. «Balgat Katlıamıyla ilgili olarak bir ülkücü yakalandı » (Un idéaliste arrêté dans l'affaire du massacre de Balgat), Cumburiyet, 15 août 1978, pp. 1, 5 ; «Balgat katliamı sanıkları savcıya, silahlı eylem için örgüt kurduklarını açıkladılar » (Les inculpés pour le massacre de Balgat expliquent au procureur qu'ils ont fondé l'organisation pour réaliser des actions armées), Cumburiyet, 12 octobre 1978, pp. 1, 9 ; T. C. Ankara - Çankır - Kastamonu Illeri

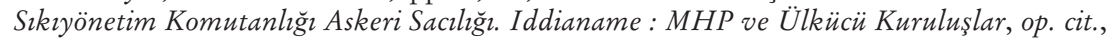
pp. 308-309. 
façons dont les ressources collectives mises en œuvre par les organisations idéalistes ont orienté certains militants vers les activités de violence. Les difficultés inhérentes au travail de terrain nous ont contraint à évacuer l'entretien comme mode privilégié d'administration de la preuve. En effet, il est quasiment impossible d'accéder aux récits de vie des anciens activistes. En situation d'entretien, ils refusent de reconnaître toute activité illégale ou non conventionnelle. En un sens, les propos recueillis témoignent de la volonté collective de faire taire le stigmate "fasciste » qui colle au parti et de réhabiliter son passé. En outre, si les activistes se racontent peu, le récit de vie et l'entretien semi-directif exposent aux mises en cohérence rétrospectives des expériences, dont on redoute la fréquence lorsqu'ils interviennent plus de trente ans après la séquence explorée. Les données recueillies en entretiens s'apparentant donc davantage à des exercices de justification des activités menées qu'à des éléments de compréhension des motivations de l'action, elles n'ont été mobilisées que ponctuellement dans la réalisation de l'enquête, lorsqu'elles permettaient de déceler des traces de subjectivation.

Nous évaluerons d'abord comment les variations des niveaux de ressources collectives opèrent sur les types et l'effectivité des dispositifs d'encadrement des activités de violence dans le Mouvement nationaliste. Nous étudierons ensuite la mise en œuvre de ces dispositifs, et la façon dont ils permettent la réalisation des activités de violence physique, par la sélection et la formation des activistes, puis par la constitution et l'entretien d'unités spécialisées dans le travail de violence.

\section{La modification des dispositifs d'encadrement des activités de violence en contexte de variation des ressources collectives du Mouvement}

La trajectoire électorale du parti, et donc l'évolution du niveau et des types de ressources dont dispose le Mouvement nationaliste, influent sur l'effectivité de ses dispositifs et sur les carrières de ses activistes. En effet, les capacités de prise en charge des activistes par le parti évoluent selon qu'il dispose d'un accès au gouvernement ou qu'il occupe une position de challenger ${ }^{18}$. Quand il accède au gouvernement, et donc aux ressources étatiques et administratives 19 centrales, le MHP est en mesure d'assurer des ressources, des prébendes, et une quasi-impunité aux activistes du Mouvement ; mais il les condamne souvent à la clandestinité et à la prison lorsqu'il est dans l'opposition. Dans ce contexte d'éloignement du parti des positions gouvernemen-

18. Selon la terminologie utilisée par Charles Tilly dans From Mobilisation to Revolution, op. cit.

19. Le terme est emprunté à Gilles Favarel-Garrigues, qui le définit comme suit : « Les ressources administratives concernent l'ensemble des agents dont les actes peuvent favoriser la réussite de l'entreprise [de prédation], qu'il s'agisse de promulguer des textes normatifs, d'octroyer des autorisations ou des dérogations, de prendre des décision budgétaires ou d'activer des services d'inspection pour déstabiliser la concurrence » («Violence mafieuse et pouvoir politique en Russie », in Favarel-Garrigues G., Briquet J-L. (eds.), Milieux criminels et pouvoir politique. Les ressorts illicites de l'État, op. cit., p. 188. 
tales, le Mouvement déploie alors des dispositifs reposant davantage sur ses ressources propres (militantes et partisanes).

\section{Les activités de violence physique en période d'accès au gouvernement}

Du 31 mars 1975 au 5 janvier 1978, le MHP participe à deux gouvernements de coalition dits de Front nationaliste. Il bénéficie alors d'une position lui permettant de profiter pleinement du système de dépouille officieux qui a cours depuis l'instauration du multipartisme en Turquie. Pendant cette période, et à partir de novembre 1979, lorsqu'il soutient depuis l'assemblée le gouvernement minoritaire formé par le parti de la justice (Adalet Partisi, AP) ${ }^{20}$, il parvient à faire nommer ses militants et ses soutiens dans plusieurs institutions d'État et entreprises publiques. Il met donc en œuvre ce qu'on peut identifier comme des activités de captation de ressources et de positions étatiques, en parvenant à intervenir dans les procédures de recrutement de la fonction publique. Ce détournement des filières d'accès au fonctionnariat aboutit à la politisation des pratiques professionnelles des agents idéalistes ${ }^{21}$, qui profitent de leurs positions pour apporter leur soutien aux membres du Mouvement. Ainsi, quand il accède au gouvernement ou y bénéficie d'une influence, le MHP s'appuie sur ces soutiens en activité dans les institutions judiciaires ou dans la police pour assurer un haut niveau d'impunité à ses militants, qui s'emploient dans la rue à tenir tête aux « communistes » ${ }^{22}$. Les positions du parti dans les arènes centrales permettent alors à des groupes d'activistes de déployer des activités de violence physique pour voir évoluer leurs positions dans les rapports de force locaux. Ici, loin de l'image d'un passage à la violence par occupation de positions marginales, c'est l'accès aux arènes étatiques centrales de l'État qui permet localement de mettre en œuvre des activités non conventionnelles d'accès aux ressources ${ }^{23}$.

Dans ce contexte, le MHP réussit donc à protéger ses activistes en intervenant dans les procédures judiciaires dans lesquelles ils sont impliqués, ou directement auprès des commissariats. En cas de fermeture par la justice de sections locales d'organisations idéalistes, les cadres du parti utilisent les réseaux constitués dans l'administration provinciale pour invalider la décision.

20. Le Parti de la justice est le principal parti de centre-droit entre 1960 et 1980 . Il est un allié électoral du MHP depuis 1975, quand il lui propose de participer à la coalition gouvernementale de Front nationaliste, alors qu'il ne dispose que de 3 sièges de députés à l'Assemblée nationale.

21. Gourisse B., « Pluralité des rapports aux normes professionnelles et politisation des pratiques dans la police turque des années 1970 », op. cit.

22. On peut considérer que l'augmentation du nombre d'altercations entre idéalistes et militants des groupes d'extrême gauche à partir de 1975 est encouragée par le MHP afin de maintenir un climat de tension sociale dans lequel il espère apparaître comme la seule solution face aux troubles causés par les « communistes ».

23. Les organisations clandestines armées d'extrême gauche, qui ne disposent jamais d'un accès direct aux ressources gouvernementales, ne bénéficient pas des mêmes possibilités d'encadrement du travail de violence. 
C'est ainsi qu'en février 1978, le responsable de la section d'Izmir obtient l'aide du préfet de province après la fermeture de la première chambre des foyers idéalistes de Bornova ${ }^{24}$. Mise à part la période pendant laquelle le CHP est au gouvernement (janvier 1978-novembre 1979), lorsque des militants idéalistes sont arrêtés par la police à la suite d'une opération armée, les organisations locales du Mouvement contactent des cadres du parti (dirigeants ou élus) qui, par l'entremise d'un ministre MHP ou d'un parti de la coalition gouvernementale, les font libérer ${ }^{25}$. Ömer Tanlak, un activiste ankariote, décrit la façon dont il a été remis en liberté après avoir été arrêté pour la fusillade d'un café situé à İsmetpaşa, à Ankara :

«Le lendemain, nous sommes allés en ville. Une recherche était menée dans les cafés, et une arme a été trouvée sur un ami, que l'on appelait Hikmet le militaire (Asker Hikmet). La police nous a réunis et nous a fait monter dans un véhicule. Avant de monter, Hikmet le militaire a dit au propriétaire du café, qui était maire ${ }^{26}$, “préviens Necati Paşa” [il s’agit de Necati Gültekin, le secrétaire général du parti]. Ils nous ont amenés au commissariat d'Anafartalar. Deux heures plus tard, nous étions libérés. ${ }^{27}$ »

Le même Tanlak, lorsqu'il évoque ses activités militantes pendant sa scolarisation au lycée de Etlik à Ankara, donne quelques informations sur le soutien que lui et ses amis trouvaient auprès du directeur du lycée, alors membre de l'association idéaliste de l'enseignement secondaire Ülkü-Bir. Il affirme notamment que pendant l'année scolaire 1976-1977, ils lui confiaient leurs armes avant d'entrer dans le lycée pour les récupérer à la fin de la journée 28 . Parfois, le parti s'assure de soutiens plus élevés dans l'administration. Des notes prises par Necati Gültekin, le secrétaire général du MHP, dans son agenda, montrent ainsi qu'un chef de la sûreté (Emniyet Müdürü) a été remercié lors d'un conseil de la présidence du MHP pour sa médiation dans l'autorisation de port d'arme accordée à des militants du parti ${ }^{29}$. Cette implantation du parti dans l'État lui permet donc d'assurer une certaine impunité à ses militants les plus actifs dans la préparation et la réalisation d'actions violentes. C'est en cela que les positions du parti dans les rapports de forces politiques nationaux orientent les pratiques des activistes au niveau local. Elles fonction-

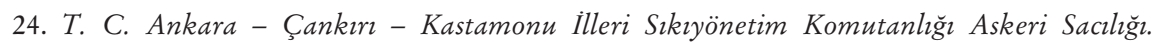
Iddianame : MHP ve Ülkücü Kuruluşlar, op. cit., p. 97.

25. En règle générale, un cadre du parti s'entretient directement avec le commissaire chargé de l'enquête. Tanlak Ö., Itiraf. Eski bir ülkücü MHP'yi anlatıyor (Confession. Un ancien idéaliste explique le $M H P)$, op. cit., p. 15.

26. Nous traduisons par «maire» le mot turc mubtar. En Turquie, les mubtar sont les représentants de l'État central dans les plus petites communes.

27. Tanlak Ö., Itiraf. Eski bir ülkücü MHP'yi anlatıyor (Confession. Un ancien idéaliste explique le $M H P)$, op. cit., p. 21.

28. Ibid., p. 37.

29. T. C. Ankara - Çankırı - Kastamonu Illeri Sıkıyönetim Komutanlı̆̆ı Askeri Sacılı̆̆ı. Iddianame : MHP ve Ülkücü Kuruluşlar, op. cit., p. 98. 
nent comme des ressources centrales permettant d'abaisser les coûts d'entrée et de maintien dans des activités illégales et non conventionnelles.

En période d'accès aux activités de captation des ressources et des positions étatiques, grâce aux soutiens qu'elles rencontrent auprès des membres des administrations provinciales, et selon les configurations locales, les sections du parti et des associations idéalistes trouvent donc l'opportunité de mettre en œuvre des activités de violence physique afin de faire évoluer les rapports de forces sectoriels (politiques, syndicaux, économiques) et locaux. Les initiatives locales des militants idéalistes en matière d'activités armées bénéficient alors du soutien du préfet ou du sous-préfet de province, de commissaires de police, ou de fonctionnaires du rang. Quand des obstacles se dressent à leurs activités politiques, les sections locales du Mouvement idéaliste peuvent alors militariser leurs répertoires d'action, assurées que des représentants du parti, élus ou fonctionnaires, interviendront en leur faveur auprès des autorités compétentes en cas d'imprévu. Ainsi, lorsqu'Abdullah Çatl1 ${ }^{30}$, alors numéro deux de l'organisation de jeunesse idéaliste, est arrêté en 1978 après l'assassinat de sept membres d'un parti d'extrême gauche à Ankara, il est rapidement libéré et obtient de faux papiers par l'entremise de fonctionnaires haut placés ${ }^{31}$.

\section{Les activités de violence physique face à la baisse des ressources collectives}

Lorsqu'il perd ses positions gouvernementales, ou lorsque ses possibilités de négocier avec le parti au pouvoir disparaissent, le MHP n'est plus en mesure d'assurer à ses activistes l'impunité dont ils bénéficiaient. Le Mouvement parvient néanmoins à compenser en partie la perte des ressources dont l'accès était assuré par ses activités de captation, et à maintenir un niveau élevé d'activités de violence.

30. Abdullah Çatlı apparaît d'abord dans les organes légaux des Foyers de l'idéal puis devient l'un des principaux organisateurs des activités de violence du Mouvement nationaliste à Ankara. Il est mêlé à l'attentat contre le pape à Rome en 1981. Il aurait ainsi fourni l'arme utilisée par son auteur, Mehmet Ali Ağça, également membre du MHP, qu'il avait auparavant aidé à s'évader de la prison où il purgeait une peine pour avoir assassiné en 1979 le rédacteur en chef du grand quotidien Milliyet. Pendant les années 1980, il est incarcéré en France, puis en Suisse pour trafic d'hérö̈ne avant de s'évader en 1990. Il est ensuite recherché par Interpol jusqu'au 3 novembre 1996, date à laquelle il périt dans un accident de voiture, dans la ville de Susurluk. Cet événement fera date dans l'histoire récente de la Turquie, puisqu'il permet d'attester les collusions existantes entre milieux criminels et secteurs étatiques. Le corps de Çatl est retrouvé en compagnie de celui de sa compagne, mais également de celui d'Hüseyin Kocadağ, un haut responsable de la police, directeur de l'école de police d'Istanbul, et de Sedat Bucak, seul survivant de l'accident, chef d'une des plus puissantes tribus de la province d’Urfa, proche du président de la République Süleyman Demirel et député d’Urfa du DYP, parti au pouvoir au moment de l'accident.

31. Elise Massicard indique que se dessinent alors « des collusions entre fonctionnaires - y compris de sécurité - et militants ayant recours à la force, collusions fondées sur des proximités politiques, en particulier de droite ». Massicard E., « Les bandes "en uniforme” en Turquie », in Favarel-Garrigues G., Briquet J-L. (eds.), Milieux criminels et pouvoir politique. Les ressorts illicites de l'État, op. cit., p. 74. 
Augmentation des cô̂ts des activités de violence physique et baisse des ressources collectives. Pendant l'année 1978, la presse relate un nombre croissant d'arrestations d'activistes idéalistes, et ce pour deux raisons. D'une part, les renouvellements d'une partie considérable des personnels des institutions judiciaires et de la direction de la sûreté (dont dépend la police), ainsi que l'aversion déclarée du CHP au gouvernement pour les idéalistes - le Premier ministre Ecevit a été plusieurs fois la cible d'attentats idéalistes lors de sa campagne électorale pour les élections législatives de 1977 - provoquent une multiplication des enquêtes et des procès dirigés contre les idéalistes. D’autre part, une fois dans l'opposition, le Mouvement nationaliste agit comme s'il désirait acquérir une position monopolistique dans le camp «anti-communiste». En multipliant les actes de violence contre les organisations d'extrême gauche, ses membres alimentent le climat d'insécurité qui règne dans le pays, ce qui permet aux leaders du parti de dénoncer l'incapacité du gouvernement à maintenir l'ordre. On peut alors considérer que les organisations idéalistes intensifient leurs activités de violence alors même que les risques encourus sont plus importants qu'en période d'accès au pouvoir ${ }^{32}$. Localement, le renouvellement d'une partie importante de l'administration déconcentrée de l'État prive les sections locales du Mouvement du soutien qu'elles pouvaient trouver auprès des fonctionnaires mutés et des personnels congédiés.

À partir du mois de mars 1978, les quotidiens nationaux relatent la multiplication des actions armées et des arrestations d'idéalistes. Les enquêtes ouvertes pour l'occasion permettent d'attester que des groupes d'activistes sont formés par des cadres du MHP, des syndicats et des associations idéalistes ${ }^{33}$. Un groupe de « commando » en activité à Ankara explique par exemple aux inspecteurs comment les présidents de section de l'ÜGD ${ }^{34}$ et de l'organisation de jeunesse du MHP leur fournissent les armes nécessaires aux opé-

32. Il est fort probable que les militants aient clairement eu conscience de l'augmentation des risques encourus lorsque le CHP est arrivé au pouvoir. Les déclarations du gouvernement, et notamment du ministre de l'Intérieur dans la presse, ainsi que l'influence que gagne le syndicat de police de gauche à la Direction de la sûreté se traduisent rapidement par la multiplication des arrestations et un « harcèlement » des idéalistes par les forces de l'ordre.

33. Le 7 août 1978, trois idéalistes, dont un président de section locale de l'ÜOD, tirent depuis une voiture sur un autobus municipal, tuent deux personnes et en blessent quatorze autres dans le quartier de Mamak à Ankara, et le 18 septembre, le président de la section de jeunesse du MHP de Şişli à Istanbul est arrêté avec trois comparses et accusé du meurtre de sept personnes, puis condamné à mort pour participation à treize différentes opérations armées. «Belediye otobüsüne ateş aşan ülkücüler 2 yurttaşı öldürüldü » (2 concitoyens ont été tués par des idéalistes qui ont ouvert le feu sur un autobus municipal), Cumburiyet, 8 août 1978, pp. 1, 4 ; «MHP Şişli İlçe Gençlik Kolu Başkanı ve 3 arkadaşı 7 kişiyi öldürmekten sanık olarak tutuklandı » (Le président de la section de jeunesse du MHP de Şişli et 3 de ses amis ont été arrêtés et inculpés du meurtre de 7 personnes), Cumburiyet, 19 septembre 1978, pp. 1,9; «13 ayrı suça katılan MHP Şişli İlçe Gençlik Kolu Başkanı ve arkadaşı idam cezası ile yargılanacak " (La peine de mort va être demandée contre le président de la section de jeunesse du MHP $d u$ district de Şişli et ses amis, pour participation à 13 différentes affaires), Cumburiyet, 11 octobre 1978, pp. 1, 9.

34. L'Association de la jeunesse idéaliste (Ülkücü Gençlik Derneği, ÜGD) succède à l'ÜOD, évoquée plus haut, lors de sa fermeture ordonnée par la justice en 1978. 
rations planifiées dans les quartiers de Beykoz et de Maltepe ${ }^{35}$. Le 27 octobre 1978, le vice-président du MHP à İskilip est arrêté en possession de deux pistolets, des fusils et des bâtons de dynamite ${ }^{36}$. En juillet 1979 , c'est le président de la confédération syndicale idéaliste MISK qui est arrêté, ainsi que plusieurs de ses cadres. La confédération est accusée de participer à la confection de bombes utilisées dans certains attentats ${ }^{37}$. En janvier 1980, les faits sont avérés lorsque le président de la section MISK de Denizli a les mains arrachées lors de la confection d'un engin explosif 38 .

Les dispositifs collectifs de maintien des activités de violence. Cette modification des positions du parti au niveau national multiplie les risques encourus par les activistes idéalistes. Mais en l'absence des dispositifs qui garantissaient l'impunité à leurs activistes, les organisations du Mouvement élaborent ce qu'on peut identifier comme des dispositifs internes d'encadrement tendant à réduire les opportunités de désengagement ${ }^{39}$, qu'on peut supposer plus nombreuses lorsque les risques liés à la mobilisation augmentent. Les organisations idéalistes soutiennent ainsi leurs activistes aux prises avec la justice, veillent à l'entretien de leur famille, et menacent de sanction toute tentative de défection.

Dès 1977, le Mouvement met en place un bureau juridique (Hukuk masası) afin de soutenir les idéalistes emprisonnés et leur famille. Ce bureau a pour mission de fournir les expertises juridiques et judiciaires utiles aux idéalistes, de préparer les procès avec les avocats qu'il rétribue, d'approcher les membres des tribunaux dans lesquels des idéalistes sont jugés afin d'acheter leur relaxe, de fournir la nourriture et les vêtements aux militants emprisonnés, et de subvenir aux besoins des familles des individus tués ou emprisonnés. En outre, le bureau est chargé d'organiser la clandestinité des idéalistes recherchés par la police en les logeant dans des appartements qu'il loue dans les grandes villes du pays. Il fournit aux prisonniers des vêtements, du linge de lit, des livres, de la nourriture et toutes autres choses destinées à améliorer leur

35. « Sanık komandolar Maltepe'de silâhları ÜGD, Beykoz'da da MHP Gençlik Kolu Başkanlarından aldıklarını anlattılar " (Les membres des commandos inculpés expliquent qu'ils obtiennent leurs armes des présidents de l'ÜGD et à Beykoz également du président de la section de jeunesse du MHP), Cumburiyet, 22 octobre 1978, pp. 1, 9.

36. "İskilip MHP II. Başkanı 2 tabanca, tüfek ve dinamitlerle yakalandığı bildirildi » (Annonce de l'arrestation du vice-président du MHP de İskilip avec deux pistolets, des fusils et de la dynamite), Cumburiyet, 27 octobre 1978, p. 5.

37. "MISSK'te bomba yapıldığ saptand1, MHP binası arand1 " (Il a été montré que des bombes sont confectionnées à MiSK, des recherches ont été menées au MHP), Cumburiyet, 19 juillet 1979, pp. 1, 11 ; « MíSK genel başkanı ile 2 yönetici sıkıyönetimce gözaltına alındı » (Garde à vne par l'état de siège du président général de MiSK et de deux de ses cadres), Cumburiyet, 21 juillet 1979, p. 6.

38. "Denizli MISKK Bölge Başkanı bomba yaparken elleri koptu, Istanbul'da 5 yere patlayıcı madde atıld1 " (Le président du MiSK à Denizli perd ses mains en confectionnant une bombe, à Istanbul, des matières explosives sont lancées dans cinq lieux différents), Cumburiyet, 4 janvier 1980 , p. 7.

39. Sur la notion de « désengagement », voir notamment : Fillieule O. (ed.), Le Désengagement militant, Paris, Belin, 2005. 
quotidien ${ }^{40}$, dont une somme mensuelle moyenne, certes modeste, de 190 Livres turques ${ }^{41}$. Le restaurant Burçak, sorte de centrale de préparation et de distribution de repas, livre quotidiennement les repas des prisonniers ankariotes.

Le Mouvement œuvre également pour que les séjours en prison soient davantage un moment d'entretien de l'engagement qu'une opportunité de défection. Souvent regroupés selon leurs affinités politiques dans des cellules collectives par l'administration pénitentiaire, les prisonniers idéalistes doivent assister à des séances de lecture, organisées par les prisonniers désignés par le Mouvement. Le parti s'assure que des séances d'apprentissage de prières, de lectures et d'explications du Coran soient organisées dans les cellules. Les prisons sont d'ailleurs considérées comme des lieux propices à la formation. Elles servent à l'instruction politique et religieuse, théorique et pratique des militants 42 .

Le Mouvement met enfin en œuvre tout un travail de valorisation de la mémoire des idéalistes morts pour la cause, qui entrent au panthéon des « martyrs » (şehit) nationalistes. Les idéalistes blessés, ainsi que les familles de ceux qui ont perdu la vie reçoivent la visite régulière de délégations de sections idéalistes locales. Ces représentants s'assurent alors que les familles concernées disposent d'assez de moyens pour subvenir à leur besoin. Si ce n'est pas le cas, ils sont censés trouver un emploi aux hommes de la famille, dans les entreprises et les commerces de sympathisants. S’ils constatent que le chef de famille souffre d'une infirmité l'empêchant de travailler, ils doivent lui fournir une « allocation d'invalidité » et en avertir le siège du parti ${ }^{43}$.

Tous ces dispositifs contribuent au maintien de l'engagement des activistes en rendant les coûts de la trahison, de la défection ou du non-conformisme très élevés. Les activistes sont totalement dépendants du parti, qui les prend en charge financièrement, les déplace, leur fournit logement, rémunération et protection. En cas de défection cette prise en charge prend fin, et les « démissionnaires » ne bénéficient plus de la bienveillance du Mouvement. Ils savent que toute tentative de désengagement ou de trahison sera sanctionnée par le groupe. Dans une phrase devenue célèbre, Türkeş, le président du MHP, lance à ses troupes : " abattez celui qui abandonne la cause, fut-ce moimême » 44 . Les cas de passages à tabac et d'assassinats perpétrés à l'encontre d'activistes en rupture attestent des risques encourus par les démission-

40. T. C. Ankara - Çankırı - Kastamonu Illleri Sıkıyönetim Komutanlı̆̆ı Askeri Sacılığı. Iddianame: MHP ve Ülkücü Kuruluşlar, op. cit., p. 183.

41. Ce qui, en mars 1978, représente 7,60 dollars. À titre de comparaison, un litre d'essence ordinaire coûte 17 livres et un paquet de cigarettes turques 15 livres en mars 1979.

42. Poulton H., Top Hat, Grey Wolf and Crescent. Turkish Nationalism and the Turkish Republic, Londres, Hurst and Company, 2006, p. 156.

43. T. C. Ankara - Çankırı - Kastamonu Illeri Sıkıyönetim Komutanlığı Askeri Sacılığı. Iddianame : MHP ve Ülkücü Kuruluşlar, op. cit., p. 105. 
naires ${ }^{45}$. Tanlak, qui abandonne la carrière d'activiste à la fin de l'année 1978, témoigne très rapidement devant la presse de ses activités illégales et désigne les personnes dont il a le plus à craindre. On ne peut s'empêcher de voir dans la médiatisation de ses activités passées un certain empressement à témoigner pour se protéger de ses anciens camarades.

On peut ainsi considérer que les organisations du Mouvement nationaliste ont su mettre en place des dispositifs permettant de diminuer les opportunités de désengagement de leurs activistes en période de baisse des ressources collectives. Ils fonctionnent comme autant d'encouragements ou d'injonctions à maintenir l'engagement dans les activités de violence encadrées par le collectif. On comprend alors que les variations des types et des niveaux des ressources collectives (administratives, étatiques ou partisanes, centrales ou locales) obligent les collectifs à modifier leurs dispositifs censés encadrer les activités de violence physique, s'ils désirent maintenir de telles activités.

L'analyse des voies par lesquelles Ömer Tanlak et Mustafa Pehlivanoğlu deux idéalistes dont les parcours d'activistes peuvent être retracés, s'engagent et se maintiennent dans des activités de violence - nous permettra d'évaluer la façon dont agissent ces dispositifs collectifs sur les individus.

\section{Les dispositifs en action : organisation et usages des activités de violence physique dans le Mouvement nationaliste}

\section{La sélection des activistes}

La sélection des activistes par le Mouvement s'effectue sur la base d'affinités idéologiques et de compétences, ou de prédispositions, à l'action violente ${ }^{46}$. La façon dont Mustafa Pehlivanoğlu, originaire du quartier de Bahçelievler à Ankara, devient activiste, correspond à ce cas de figure. Il s'inscrit en 1976 aux cours de Taekwondo de son quartier. La salle de sport se situant à proximité du siège du MHP, ce sont de jeunes militants idéalistes qui animent les activités sportives. Lors de ses entraînements, Pehlivanoğlu se socialise aux milieux idéalistes du quartier. Il est rapidement convié à prendre part à des expéditions menées contre des groupes de militants d'extrême

44. Bozarslan H., « Le phénomène milicien, une composante de la violence politique dans la Turquie des années 1970 », Turcica, Vol. 31, 1999, p. 209.

45. Lorsqu'un groupe composé d'une cinquantaine de jeunes membres des sections de jeunesse d'Izmir quittent le parti en 1969 afin de créer le groupe Développement énergique de l'activité nationale (Nasyonel Aktivite Zinde Inkişaf, N.A.Z.I), ses membres sont passés à tabac par de jeunes membres du MHP et de l'Union des foyers de l'idéal d'Izmir. Feyizoğlu T., Firtınalı Yillarda Ülkücü Hareket (Le mouvement idéaliste dans les années de tempête), İstanbul, Ozan Yayınc1lık, 2000, p. 32.

46. La division du travail qui a cours au Mouvement nationaliste comporte une dimension genrée. Elle réserve aux hommes la mise en œuvre des activités de violence. Les femmes, rassemblées dans des organisations spécifiques, participent généralement à l'organisation d'activités culturelles et folkloriques. 
gauche, et se bat à plusieurs reprises jusqu'à son arrestation et sa condamnation pour coups et blessures à une peine de vingt et un mois de prison. Six mois après le début de son incarcération, il reçoit la visite d'İsa Armağan et d'Abdullah Çatlı, deux cadres de l'organisation de jeunesse idéaliste d'Ankara spécialisés dans l'organisation d'activités armées. Ils l'informent qu'ils ont réussi à obtenir sa libération par l'intermédiaire de soutiens du parti en poste dans l'administration judiciaire ${ }^{47}$, et lui proposent de participer aux activités qu'ils organisent. Il est alors libéré, logé dans une « maison de célibataires » idéalistes du quartier ankariote de Karapınar, rémunéré, et participe à des opérations organisées par son unité de quartier. Ces petites unités spécialisées dans les activités de violence physique sont créées par les associations de jeunesse idéalistes, en coordination avec les sections de jeunesse du MHP. Elles sont composées d'individus repérés lors des altercations avec l'extrême gauche, ou dans les clubs de sports de combat ouverts par des sections locales du Mouvement. Ömer Tanlak, l'autre activiste ankariote à propos duquel nous disposons d'informations, dit s'être rapproché d'une organisation de jeunesse idéaliste dès son entrée au lycée, lors des altercations régulières qui éclataient entre lycéens nationalistes et "gauchistes ». Il est convié par des militants du lycée à assister aux séminaires organisés au foyer idéaliste du quartier d'Etlik, puis rapidement intégré à une petite équipe de frappe ${ }^{48}$. Se réalise ainsi ce qu'on peut identifier comme un travail collectif de sélection des activistes du Mouvement, qui passe notamment par le repérage des individus présentant des dispositions aux activités de violence physique, une motivation, ou des compétences spécifiques à ce type d'engagement.

\section{La formation des activistes}

Lors de leur entrée dans le groupe, les futurs activistes sont l'objet du même travail de normalisation institutionnelle que l'ensemble des militants du Mouvement. Dès la reprise en main du parti en 1965 par Alparslan Türkeş, ancien colonel de l'armée turque, les cadres du parti travaillent ainsi à la diffusion d'une esthétique militaire au MHP et dans l'ensemble des organisations du Mouvement. Le niveau de discipline est très élevé et organisé autour d'un système d'examen collectif reposant sur l'envoi régulier de rapports au siège du parti, que chaque échelon des organisations idéalistes prépare sur les membres des échelons inférieurs. Les "simples » militants sont condamnés à exécuter les ordres sous peine d'être victime d'ostracisme ou de recevoir de sévères corrections. C'est ce que suggère Remzi, un enquêté rencontré à Ankara, lorsqu'il affirme : "nous étions des robots, les ordres arrivaient et on les respectait 49 ».

47. T. C. Ankara - Çankır - Kastamonu İlleri Sıkıyönetim Komutanlı̆̆ı Askeri Sacılı̆̆ı. Iddianame : MHP ve Ülkücü Kuruluşlar, op. cit., p. 314.

48. Tanlak Ö., Itiraf. Eski bir ülkücü MHP'yi anlatyyor (Confession. Un ancien idéaliste explique le $M H P)$, op. cit., p. 14.

49. Entretien semi-directif réalisé avec Remzi à Ankara le 3 juillet 2008. 
Dès la fin des années soixante, les organisations idéalistes mettent également en place des dispositifs spécifiques destinés à la formation à l'action violente. À partir de l'été 1968, les organisations du Mouvement nationaliste organisent des « camps de commandos » dans différentes provinces turques afin d'assurer une formation idéologique et physique aux militants en charge des activités de violence dans le Mouvement. Un rapport remis au Premier ministre Süleyman Demirel en 1970 évoque l'existence de vingt-huit « camps » dans toute la Turquie entre 1968 et 1970. Leur organisation est calquée sur le modèle de la caserne militaire. Ils accueillent des groupes composés de dix à cent cinquante jeunes militants, pendant une période de quinze à vingt jours. La journée type dans un « camp de commando » se déroule comme suit 50 :

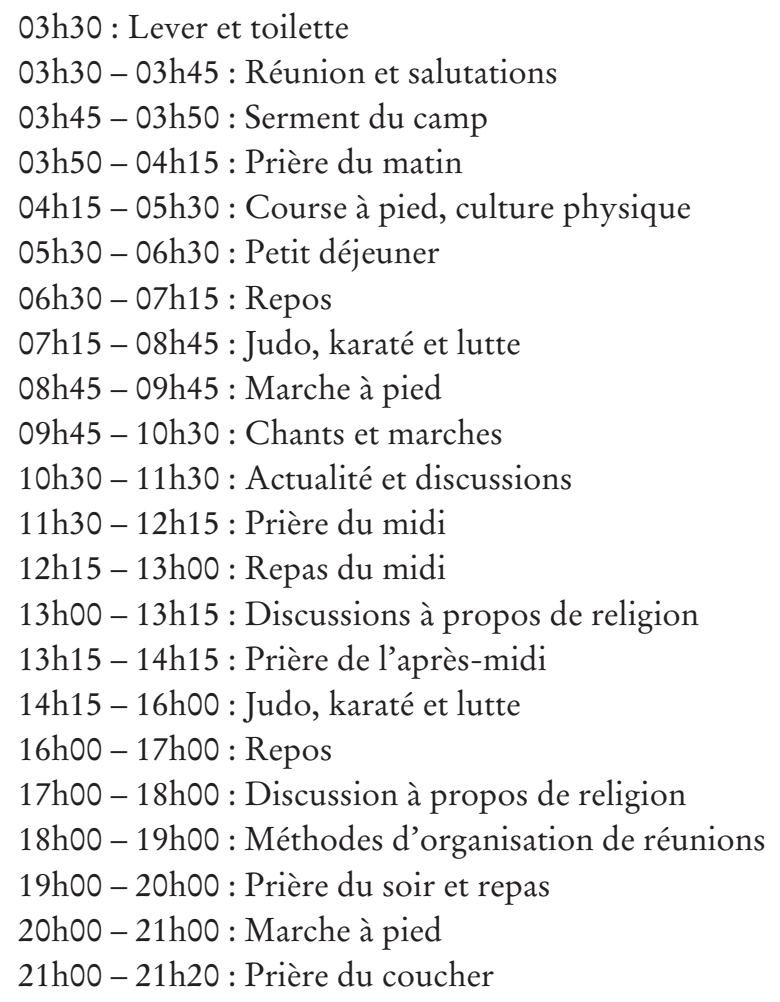

L'existence de ce type de camp n'a jamais été reconnue par le MHP, mais selon Mehmet Şandır, cadre de la section stambouliote de l'association de jeunesse idéaliste avant 1980 :

«Il y avait des camps de commandos, mais pas de violence du tout,

50. Ülkücü Komando Kamplar. AP Hükümeti’nin 1970'te Hazırlattığ MHP Raporu (Les camps de commandos idéalistes. Le rapport qu'a fait préparer le gouvernement AP en 1970), Istanbul, Kaynak yayınları, 1978, p. 20. 
non non... C'étaient des camps... comme des camps de scouts, avec du sport, de la marche, des séminaires... On n'a jamais rien fait contre l'État, ce sont les communistes qui étaient contre l'État. 51 »

Si ces camps sont réservés à une minorité de militants 52 - probablement quelques centaines - les dispositifs qu'ils mobilisent visent à la diffusion et à l'inculcation des valeurs du MHP, articulées autour de l'esthétique militaire (repérable par le format même du camp), de la religion (par les cinq prières quotidiennes et les discussions sur la religion), de la culture physique (marche à pied et sports de combat) et de l'activité politique (par les discussions sur l'actualité et l'acquisition d'un savoir-faire organisationnel). Tous les activistes ne bénéficient pas d'une formation en camp de commando. Le plus souvent, ils s'entraînent dans des salles de sports de combat du Mouvement, ils sont conviés à des excursions en forêt ou à la campagne, pendant lesquelles ils sont initiés en petits groupes au tir à l'arme à feu. C'est ce type de «formation » que reçoit Tanlak lors de son entrée dans le foyer de Etlik :

«Nous n’avions pas de professeur spécifique pour l'apprentissage $\mathrm{du}$ maniement des armes et des bombes. Ceux qui s'y connaissaient apprenaient à ceux qui ne savaient rien. Ils nous ont dit où ils avaient appris. Selon leurs dires, c'est auprès d'Abdurrahman Sağkaya, militant et directeur général d'Antbirlik 53 en 1977, qu'ils ont appris ${ }^{54}$. »

Les camps de commandos s'insèrent donc dans un ensemble de dispositifs d'encadrement des activistes. Le format des camps, calqué sur celui de la caserne, la forte structuration hiérarchique et pyramidale du mouvement idéaliste, ainsi que l'existence de règles de vie et de normes de comportement contraignantes dont le respect est contrôlé par chacun, encouragent ainsi à un haut niveau de conformité des activistes aux relations de pouvoir internes au mouvement.

51. Entretien semi directif réalisé avec Mehmet Şandir à Ankara, le 5 avril 2007.

52. La structure organisationnelle du Mouvement nationaliste, ainsi que les dispositions légales relatives à l'inscription dans un parti politique en Turquie dans les années soixante-dix rendent vain tout travail de comptabilisation des membres du mouvement. Ainsi, il est interdit aux fonctionnaires de participer aux activités de partis politiques. Par ailleurs, les appartenances des militants sont souvent multiples, ces derniers étant parfois membres du parti, d'une organisation professionnelle ou d'un syndicat idéaliste, d'une association culturelle, etc. Il faut également noter que tous les militants ne paient pas systématiquement leur cotisation, ou ne sont pas formellement inscrits dans le Mouvement. En outre, les sections locales limitent volontairement le nombre officiel de leurs membres, afin de ne pas avoir à se plier aux dispositions légales des lois sur les partis politiques et les associations réglant la vie interne des organisations.

53. Antbirlik est une entreprise publique située à Antalya, dont le directeur a été nommé grâce à l'entremise du MHP alors au gouvernement.

54. Tanlak Ö., İtiraf. Eski bir ülkü̈ü̈ MHP'yi anlatıyor (Confession. Un ancien idéaliste explique le $M H P)$, op. cit., p. 14. 
Outre ce travail de sélection et de formation de leurs activistes, les organisations idéalistes planifient et organisent les activités de violence. Dans les grandes villes du pays, des bibliothèques de quartier sont créées, dont la véritable fonction est de servir de quartiers généraux pour les groupes armés locaux. Ce sont des centres où sont basées les équipes de frappe et où l'argent est collecté afin de prendre en charge les frais quotidiens de leurs membres. Les idéalistes filent la métaphore jusqu'à adopter tout un langage codé consistant à utiliser des titres de livres pour désigner des types d'armes, de munitions ou de matières explosives 55 .

Une partie de l'organisation des activités armées et clandestines est directement prise en charge par les organes centraux du MHP. Un document, préparé par le bureau du vice-secrétaire général du MHP, atteste de l'implication des cadres du parti en ce domaine. Après avoir rappelé que « le travail illégal et clandestin est une obligation » pour le Mouvement, le document énonce les « règles » qui doivent être respectées dans son organisation:

«(1) Toutes les activités doivent être organisées avec le minimum d'hommes possible. [...]

(2) Les militants 56 ne doivent pas connaître les raisons pour lesquelles un autre militant a été choisi. Un militant doit savoir tout ce qu'il doit savoir pour réaliser ce pour quoi il a été choisi, mais à part cela, il ne doit rien savoir de plus sur la nature de l'opération. [...]

(3) Le militant nationaliste doit être dans une discipline telle qu'il ne doit pas chercher à en savoir plus que ce sur quoi portent ses propres opérations. [...]

(4) Les militants qui prennent part à une opération illégale doivent être séparés des militants avec lesquels ils ont l'habitude de discuter et être placés dans un autre environnement. [...]

(5) Le militant doit savoir vivre au milieu du peuple. Il doit veiller à se comporter de façon appropriée à la vie dans un village, ou dans une ville. [...] S'il est recherché par la police ou s'il est sous le coup d'une décision de justice, il doit se cacher et vivre de façon clandestine. ${ }^{57}$ "

55. Le livre Dış Politika ve Kıbrıs désigne de la dynamite, Türkiye Taribi une arme automatique de gros calibre, Gönül Seferberliği une arme de calibre $14 \mathrm{~mm}$, Köse Kadı une arme de calibre $9 \mathrm{~mm}$, Büyük Türkiye (écrit par Dündar Taşer) une arme de calibre 7,65 mm, Konuşmalar un Smith et Wesson, Leninizm-Komünizm une arme de calibre 6,35 mm, Temel Görüşler une arme automatique de petit calibre, Diğerleri (les autres) une arme automatique en général. $T$. C. Ankara-Çankırı-Kastamonu Illeri Sıkıyönetim Komutanlı̆̆ı Askeri Sacıllğı. Iddianame : MHP ve Ülkücü Kuruluşlar, op. cit., p. 285.

56. Le terme «militan » est utilisé en langue turque pour désigner les militants activistes.

57. T. C. Ankara - Çankırı - Kastamonu Illeri Sıkıyönetim Komutanlığı Askeri Sacılı̆̆ı. Iddianame : MHP ve Ülkücü Kuruluşlar, op. cit., p. 291. 
Mustafa Pehlivanoğlu est arrêté pendant l'été 1978 après avoir participé au mitraillage de plusieurs salons de thé dans le quartier ankariote de Balgat. Il explique alors comment étaient organisées les opérations armées de la «bibliothèque » de Karapınar. Selon ses propres mots, « les ordres et les instructions venaient de l'Association du grand idéal (Büyük Ülkü Derneği, BÜD) 58 » :

«Quand une opération allait être organisée, des instructions étaient envoyées à l'organisation concernée par le foyer d'Ankara (Ankara ocă̆ $\imath$ ) et le siège de l'Association du grand idéal. Une fois que les instructions étaient transmises, des sections ou des bibliothèques fournissaient les armes. Les personnes qui devaient prendre part à l'opération se rendaient au foyer d'Ankara, prenaient les armes, puis quand ils avaient fait ce qu'ils avaient à faire, venaient les redéposer. [...] Le foyer d'Ankara faisait un rapport au siège pour chaque opération réalisée. [...] Toutes les semaines, des réunions étaient organisées au siège de la BÜD, qui rassemblaient les responsables de chaque section. [...] Comme nous, nous étions à un niveau inférieur, nous n'avions pas de contact avec le MHP ${ }^{59}$.»

Selon ses dires, Pelhivanoğlu était situé à l'échelon le plus bas de l'organisation des activités armées, ce qui est tout à fait plausible compte tenu de la façon dont il est repéré et recruté par les cadres. Au niveau supérieur se trouvait İsa Armağan, qui dirigeait le petit groupe, et assurait la liaison avec les autres organisations idéalistes. Pendant quelques mois, les activités du groupe ne dépassent pas les quartiers qui jouxtent Karapınar. Mais ses membres sont choisis pour rejoindre Çatlı, sous les ordres duquel ils sont placés, et mènent des opérations dans l'ensemble de la capitale. Pelhivanoğlu explique ainsi :

«Après quelque temps, $[\ldots]$ nous avons obtenu des fonctions au centre général. Nous sommes entrés sous les ordres de Çatlı et avons perpétré des assassinats et des agressions. Armağan prenait les instructions auprès de Çatl, nous les transmettait, et nous réalisions ensemble l'opération. Muhsin Yazıcıoğlu [le président de l'association de jeunesse idéaliste ÜGD] assurait la protection des idéalistes qui prenaient part à de telles opérations ${ }^{60}$. 》

Le témoignage de Pelhivanoğlu met en lumière l'organisation du travail clandestin armé dans le Mouvement nationaliste. Les responsables des petits

58. L’Association du grand idéal (Büyük Ülkü Derneği, BÜD) est une des premières associations

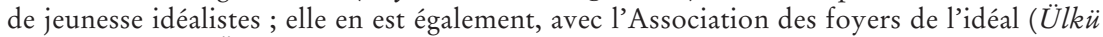
Ockları Derneği, ÜOD), l'une des plus importantes en nombre de membres.

59. T. C. Ankara - Çankırı - Kastamonu Illeri Sıkıyönetim Komutanlı̆̆ Askeri Sacılı̆g. Iddianame: MHP ve Ülkücü Kuruluşlar, op. cit., pp. 308-309.

60. Ibid., p. 312. 
groupes de quartier effectuent la liaison avec les sections locales (de district ou de province) des associations idéalistes, et ce sont leurs présidents qui assurent la connexion avec les cadres du MHP, et notamment avec les cadres chargés officieusement de fournir une partie des armes et des financements nécessaires aux activités clandestines, et parfois de transmettre les directives des organes centraux du MHP. Ce type d'organisation est repérable ailleurs dans le pays. Pour la réalisation d'opérations ciblées, il s'agit la plupart du temps de constituer des équipes de trois ou quatre personnes, de mettre des armes à leur disposition dans un "appartement de célibataire » où ils se réfugient pendant quelques jours avant de se disperser, puis laissent les armes sur place afin que d'autres militants viennent les récupérer pour les cacher dans une autre province ${ }^{61}$.

En bénéficiant du soutien de l'ensemble des organisations locales du Mouvement, les unités armées disposent des moyens logistiques nécessaires pour mener à bien leurs activités. Les membres de ces unités reçoivent ainsi un salaire régulier, généralement versé en mains propres par des cadres locaux du Mouvement ${ }^{62}$. En retour, les organisations idéalistes légales se servent de ces unités pour mener des campagnes d'intimidation, liquider physiquement les individus susceptibles d'entraver leur action, ou encore pour trouver les fonds nécessaires à leurs activités. Tanlak, l'activiste idéaliste repenti, explique ainsi à la presse comment son petit groupe était employé pour obtenir des commerçants récalcitrants les tributs demandés par les associations idéalistes légales d'Antalya ${ }^{63}$. Pour des opérations de plus grande envergure, Tanlak affirme que les directives sont transmises oralement par la section de jeunesse du MHP au président du foyer idéaliste de la province ou de la ville concernée, qui sélectionne alors les activistes et forme des équipes de frappe. Selon Tanlak, ce sont toujours les foyers idéalistes qui fournissent les moyens nécessaires à la réalisation des opérations, dont les armes et les matières explosives : « le président du foyer fournissait les matières explosives et les armes. Il appelait et disait "il y a du travail", il donnait le matériel, expliquait le plan et partait $64 »$. En règle générale, les organisations idéalistes locales transmettent directement leurs directives aux associations de jeunesse du Mouvement, sans en référer au parti. Les organisations commanditaires rétribuent alors finan-

61. Ibid., p. 345. Le faible niveau de ressources des forces de l'ordre, incapables de maintenir le calme dans un contexte d'intensification des affrontements entre groupes radicaux, explique la facilité avec laquelle le Mouvement nationaliste a pu organiser ses activités de violence physique.

62. Le financement des activités de violence peut être assuré par les associations idéalistes, qui allouent ponctuellement aux unités une partie de leurs ressources (sans toutefois le faire apparaître dans leur comptabilité), ou par leurs activités d'extorsion et de racket menées auprès des populations résidant dans les quartiers dans lesquels elles sont en position de force. Dans les cas où les groupes idéalistes exercent un contrôle milicien sur le quartier, c'est la milice elle-même qui est chargée de ces activités d'extorsion.

63. Tanlak Ö., İtiraf. Eski bir ülkü̈ü MHP'yi anlatıyor (Confession. Un ancien idéaliste explique le $M H P)$, op. cit., pp. 58-59.

64. Ibid., p. 14. 
cièrement les activistes choisis ou leur section d'affiliation. Ces activités de violence organisée permettent ainsi aux organisations idéalistes de renverser un rapport de forces local (politique, syndical, etc.) jusqu'alors à leur défaveur, par l'intimidation ou l'élimination d'individus qui entravent leurs activités.

L'étude des activités de violence physique organisées au sein du Mouvement nationaliste permet d'identifier toute une série de dispositifs mis en œuvre par les organisations idéalistes, par lesquels l'engagement et le maintien dans des activités de violence physique sont encouragés. L'analyse donne ainsi à voir un travail de sélection, de formation et d'entretien d'individus dont le Mouvement s'assure les services et la conformité dans la réalisation d'activités de violence armée organisées. Quand le parti accède au pouvoir, il dispose des ressources et des positions nécessaires pour garantir ce qu'on peut identifier comme une réduction des coûts des activités violentes (en négociant avec une partie des personnels des institutions judiciaires et du maintien de l'ordre). Mais quand il quitte le gouvernement et abandonne ses positions dans les institutions étatiques, le niveau de risque encouru par ses activistes est plus élevé. Les organisations idéalistes réussissent néanmoins à obtenir le maintien des activités de violence malgré la perte globale de leurs ressources, en déployant des dispositifs idéologiques et pratiques visant à placer leurs activistes, ainsi que leurs familles, dans une situation de subordination au groupe. Les rétributions financières, la valorisation sociale de l'activité et des risques qui lui sont liés, la prise en charge de la famille et l'accompagnement lors des procès et de l'emprisonnement ont ainsi contribué, lorsque le parti était privé de l'accès aux ressources centrales, à obtenir le maintien des activités de violence, dans les situations mêmes où celui-ci est particulièrement susceptible d'être remis en cause par les individus. 